\title{
Interaction of Trichomonas vaginalis and Tritrichomonas foetus with keratin: an important role in parasite infection
}

\author{
Ricardo Chaves Vilela', Marlene Benchimol'++ \\ 1Programa de Pós-graduação em Ciências Biológicas, Instituto de Biofísica Carlos Chagas Filho, Universidade Federal do Rio de Janeiro, \\ Rio de Janeiro, RJ, Brasil ²Universidade Santa Úrsula, Rua Jornalista Orlando Dantas 59, 22231-010 Rio de Janeiro, RJ, Brasil
}

Trichomonas vaginalis and Tritrichomonas foetus are human and bovine parasites, respectively, that provoke the sexually transmitted disease trichomoniasis. These extracellular parasites adhere to the host epithelial cell surface. Although mucinases and proteases have been described as important proteins for parasite adhesion to epithelial cells, no studies have examined the role of the keratin molecules that cornify the vaginal epithelium. Here, we investigated the interaction of $\mathrm{T}$. vaginalis and $\mathrm{T}$. foetus with human keratin in vitro; additionally, adherence assays were performed in cattle with $\mathrm{T}$. foetus to elucidate whether trichomonads were able to interact with keratin in vivo. We demonstrated that both $\mathrm{T}$. vaginalis and $\mathrm{T}$. foetus interacted directly with keratin. Additionally, the trichomonads ingested and digested keratin, shedding new light on the Trichomonas infection process.

Key words: Trichomonas vaginalis - Tritrichomonas foetus - endocytosis - keratin - parasite-cell interaction

Human trichomoniasis and bovine trichomoniasis are sexually transmitted diseases provoked by the parasites Trichomonas vaginalis and Tritrichomonas foetus, respectively. In humans T. vaginalis is capable of causing severe vaginal, ectocervical, prostatic and urethral inflammation and it is linked with sterility, pelvic inflammatory disease, adverse pregnancy outcomes, postnatal complications and cervical cancer (Petrin et al. 1998, Schwebke \& Burgess 2004, Nanda et al. 2006, Johnston \& Mabey 2008). Furthermore, $T$. vaginalis contributes to increased human immunodeficiency virus transmission rates (Rendon-Maldonado et al. 2003, Galvin \& Cohen 2004). In the beef industry, $T$. foetus infections cause economic losses. Although infection with trichomonads usually leads to undetected vaginitis, endometritis, pregnancy loss and transient infertility, it occasionally progresses to pyometra and permanent endometrial damage (Parsonson et al. 1976, BonDurant 2005). Over the last years, there has been a considerable amount of research on these parasites, their virulence factors and the host immune response.

Generally, T. vaginalis is described as a vaginal parasite. Trichomonads can interact and cross the mucus barrier on vaginal epithelia (Alderete \& Garza 1988, Lehker \& Sweeney 1999) and this might link to the clinical manifestation known as colpitis macularis or strawberry cervix (Wølner-Hanssen et al. 1989, Kassem \& Majoud 2006). However, the urogenital tract also displays some keratinised areas and the interaction between trichomonads and keratin has not been studied

Financial support: CNPq, CAPES, FAPERJ, PRONEX, AUSU

+ Corresponding author: marlenebenchimol@gmail.com

Received 24 February 2011

Accepted 16 August 2011 yet. It is not clear whether these parasites exhibit a preference for keratinised or non-keratinised areas of the vaginal epithelium. The aim of this study was to investigate the interaction of trichomonads with vaginal tissue and keratin to investigate the relationship between these parasites and keratin.

\section{MATERIALS AND METHODS}

Parasites - The JT strain of T. vaginalis was isolated at the University Hospital of the Federal University of Rio de Janeiro, Brazil; it has been maintained in culture since the 1980s. The K strain of T. foetus was isolated by Dr H Guida (Brazilian Agricultural Research Corporation, Rio de Janeiro, Brazil) from the urogenital tract of a bull. Parasites were cultivated in trypticaseyeast extract-maltose medium (Diamond 1957) supplemented with $10 \%$ foetal bovine serum. Parasites were grown for $36-48 \mathrm{~h}$ at $37^{\circ} \mathrm{C}$, which corresponds to the logarithmic growth phase.

Co-incubation and attachment assay - interaction with the vagina in vivo - To analyse in vivo interactions, $10^{4}$ T. foetus $\mathrm{K}$ cells in phosphate balanced salt solution (PBS) were injected in the vaginal canal of six cows, which were then maintained for two-four weeks post-infection. In control experiments, the parasites were omitted. The vaginas were removed in the slaughterhouse, immersed in sterile PBS containing $0.05 \mathrm{~g} / \mathrm{L}$ gentamicin sulphate and transported to the laboratory within $2 \mathrm{~h}$. Vaginal fragments were processed for scanning electron microscopy (SEM) as described below.

Attachment and endocytosis assays - in vitro interaction with keratin from human epidermis - To observe whether trichomonads interact with keratin, coverslips were immersed in a solution containing $5 \mu \mathrm{g} / \mathrm{mL}$ of human epidermis keratin (Sigma, USA) diluted in water and then incubated for $1 \mathrm{~h}$ at $37^{\circ} \mathrm{C}$. After all of the water was evaporated, $T$. vaginalis or $T$. foetus were added to the keratin-coated coverslips and allowed to interact 
for $1 \mathrm{~h}$. The assays were then processed for SEM as described below. Parasite interactions with keratin in liquid suspensions were also assayed; samples from the experiments were processed for transmission electron microscopy (TEM) as described below. To visualise keratin localisation, immunofluorescence analysis was performed using a monoclonal anti-keratin antibody as described below.

SEM - After parasite interaction, coverslips were fixed in $2.5 \%$ glutaraldehyde in $0.1 \mathrm{M}$ sodium cacodylate buffer at $\mathrm{pH} 7.2$ for $2 \mathrm{~h}$. Afterwards, the coverslips were washed with PBS, post-fixed for $15 \mathrm{~min}$ in $1 \%$ osmium tetroxide (OsO4), dehydrated in ethanol, critical point-dried with $\mathrm{CO}_{2}$ and sputter-coated with gold-palladium. The samples were examined with a JEOL 5800 scanning electron microscope.

TEM - The liquid preparation of trichomonads coincubated with keratin was fixed at room temperature with $2.5 \%$ glutaraldehyde in $0.1 \mathrm{M}$ cacodylate buffer at $\mathrm{pH}$ 7.2. Afterwards, the oviducts were washed with PBS. Post-fixation was performed for $30 \mathrm{~min}$ with $1 \%$ OsO4 in cacodylate buffer containing $5 \mathrm{mM} \mathrm{CaCl}_{2}$ and $0.8 \%$ potassium ferricyanide. Parasites were washed with PBS, dehydrated in acetone and embedded in Epon. Ultrathin sections were stained with $10 \%$ uranyl acetate and 5\% lead citrate and observed with a JEOL 1210 transmission electron microscope.

Immunofluorescence microscopy - One hour after parasite addition, the keratin-coated coverslips were washed with PBS and fixed in $4 \%$ paraformaldehyde in $0.1 \mathrm{M}$ phosphate buffer, $\mathrm{pH}$ 7.2. Next, the coverslips were incubated overnight at $4^{\circ} \mathrm{C}$ with the monoclonal mouse anti-human keratin antibody (C5992, Sigma, USA). Next, the coverslips were incubated for $1 \mathrm{~h}$ with an Alexa Fluor 488-conjugated anti-mouse antibody diluted 1:100 in bovine serum albumin/PBS. Finally, the coverslips were washed and examined using an Axiophot II Zeiss microscope equipped with ultraviolet epifluorescence; images were acquired with a chilled Zeiss CCD camera (Axiocam MRC 500) and processed using Adobe Photoshop (Adobe, USA).

Quantification analysis - The quantification of the percentage of parasites attached or unattached to keratin was achieved by counting 80 different fields of each sample to obtain a total of 200 cells both by SEM and TEM. The mean number of cytoplasmic vacuoles in keratin-attached and non-attached cells was measured by counting 60 cells using TEM. The graphics and standard deviation were built on Graphpad prism 5.

\section{RESULTS}

Interactions between T. foetus and bovine vaginal epithelial cells (VECS) - The examination of bovine vaginal mucosa samples without parasites showed highly cornified tissue with filiform papillae (Fig. 1A). After the inoculation of T. foetus, we observed that some parasites were attached to the mucus layer of the keratinised tissue (Fig. 1B).
Trichomonads interact with human keratin in vitro - To determine if parasites attach directly to living keratinised cells or over disrupted areas of the vaginal epithelium, in vitro interactions with keratin were performed. In addition, we were interested in following trichomonad attachment to keratin and the pathway and fate of this protein, if this interaction occurred. For this purpose, the trichomonads were incubated with pure human keratin in vitro. As expected, both T. vaginalis and T. foetus adhered to the purified keratin (Fig. 2); adhesion was observed both by SEM and TEM (Figs $2,3)$. Interestingly, after parasite attachment, endocytosis occurred, indicating parasite uptake of keratin (Fig. 3). Endocytosis and vacuoles containing endocytosed material (Fig. 3B-D) were observed in both of the trichomonads studied (Fig. 3). To confirm whether the material being endocytosed was keratin, immunodetection using a monoclonal keratin-specific antibody was performed. Keratin was positively labelled in several structures attached to the surface of both $T$. vaginalis (Fig. 4A-C) and T. foetus (Fig. 4D-F). Moreover, a small fragment of endocytosed keratin was observed (inserts in Fig. 4A-C).

Statistical quantification of keratin attachment and digestion vacuoles - To measure the specificity of parasite attachment to keratin, multiple experiments were analysed comparing the percentage of parasites attached to keratin to those non-attached. The results showed that more than $40 \%(\mathrm{p}<0.034)$ of the T. vaginalis population
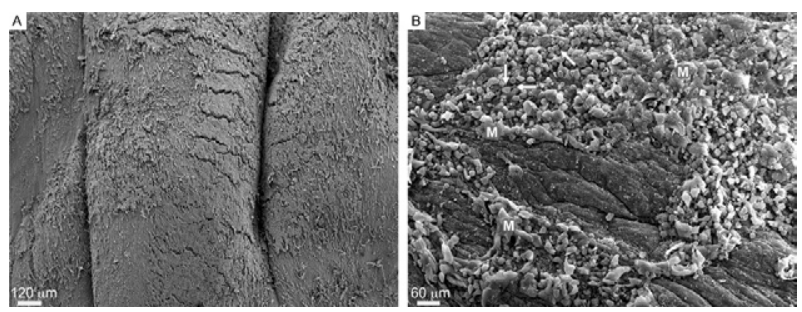

Fig. 1: scanning electron micrograph of cornified bovine vaginal tissue without parasites (A). Some Trichomonas foetus parasites (arrows in B) can be observed attached to the lumen of mucified vaginal epithelial tissue (M).
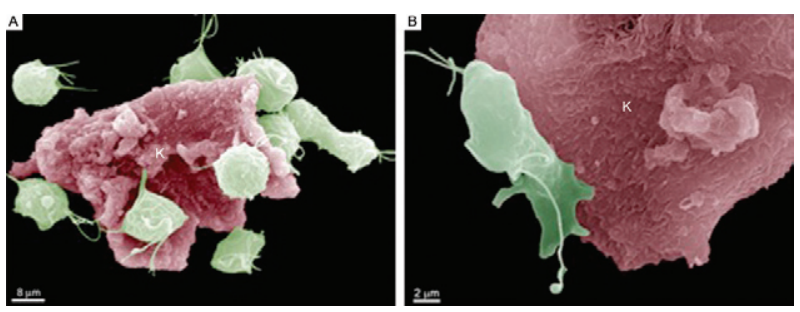

Fig. 2: scanning electron micrographs of the interaction of Trichomonas vaginalis (A) and Trichomonas foetus (B) with purified human keratin $(\mathrm{K})$. Note how several $T$. vaginalis parasites are attached to a keratin particle (A). T. foetus (B) spread in an amoeboidal shape thus increasing the surface contact with the keratin particle. 
was attached to keratin, whereas approximately $33 \%$ of the total T. foetus $(\mathrm{p}<0.036)$ parasites were found to be interacting with keratin particles (not shown). To analyse whether trichomonads have endocytosed keratin, the mean number of digestion vacuoles of keratin-attached trichomonads was compared to the vacuoles found in non-attached parasites by TEM. A two-fold increase was observed in keratin-attached vacuoles of T. vaginalis ( $\mathrm{p}$ $<0.026$ ) and the vacuoles increased by more than four times in T. foetus ( $\mathrm{p}<0.031)$ (Fig. 5).

\section{DISCUSSION}

The ability of trichomonads to colonise the epithelia of the urogenital tract has been studied in recent years; however, few studies have investigated whether preferential sites for attachment and colonisation exist.

It has been demonstrated that $T$. vaginalis cells are real scavengers and that they seem to interact and ingest a variety of cells, such as VECs, erythrocytes, lactobacilli and leucocytes (Rendon-Maldonado et al. 1998). However, their behaviour concerning the protein keratin has not been analysed. It is unknown whether the parasites select keratinised cells or a fragile area of the epithelium for attachment. Corbeil et al. (1989) studied T. foetus adhesion on isolated bovine VECs and concluded that parasites preferentially attached to squamous cells. It has also been published that the ciliated cells present in the bovine oviduct are not a preferential site for trichomonad interaction; instead, the secretory cells seem to be the adhesion site of the parasites (Midlej et al. 2009). Some authors have claimed that $T$. vaginalis exhibited an increased ratio of attachment and cytotoxicity on epithelial cells compared to fibroblasts (Alderete \& Garza 1985). However, these data were not confirmed and other studies have shown that trichomonads are capable of attaching to substrates such as laminin, fi-

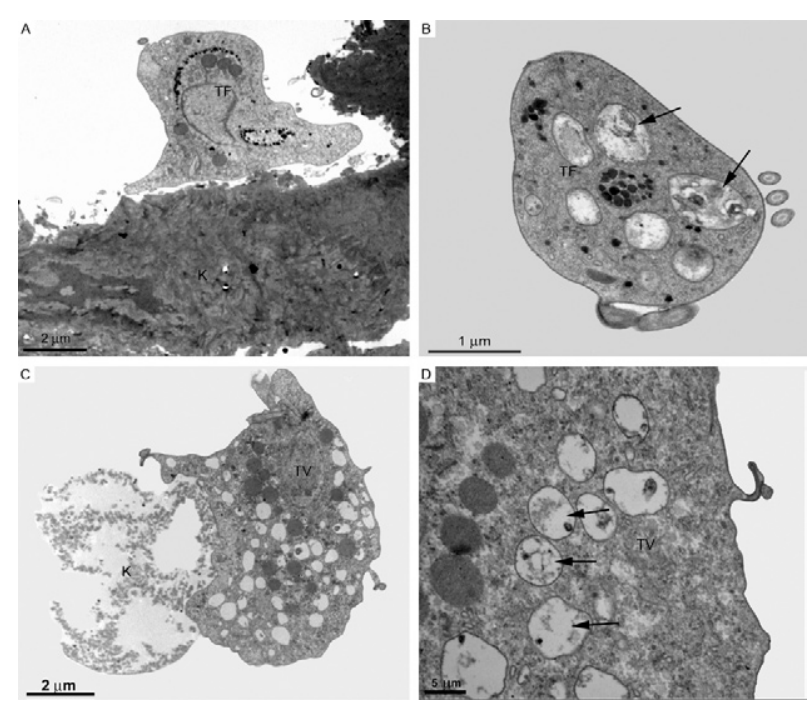

Fig. 3: transmission electron microscopy of interaction of Trichomonas foetus $(\mathrm{TF})(\mathrm{A}, \mathrm{B})$ and Trichomonas vaginalis $(\mathrm{TV})(\mathrm{C}, \mathrm{D})$ with keratin $(\mathrm{K})$ fragments. Several endocytic vacuoles containing keratin particles are observed (arrows in B, D). bronectin and collagen, which are known components of the extracellular matrix and basement membrane (Costa e Silva-Filho et al. 1988, Benchimol et al. 1990, Crouch \& Alderete 1999, Alvarez-Sánchez et al. 2000).

Before interacting with keratinised cells, trichomonads need to cross the mucous barrier found in the vagina. Trichomonads have proteolytic enzymes called mucinases that digest the mucus and allow the parasites to access to VECs (Alderete \& Garza 1988, Lehker \& Sweeney 1999). The description of a strawberry cervix in Trichomonas-positive patients (Wølner-Hanssen et al. 1989, Kassem \& Majoud 2006) led us to hypothesise that trichomonads could interact with keratin, either to gain access to deeper parts of the vaginal epithelium or to use this protein as nutrition. Moreover, an experimental study using a murine model of $T$. vaginalis infection showed parasite attachment to the mouse vaginal keratin layer and alterations in muscle tissue after extended periods of infection (Escario et al. 2009).

Taken together, our findings and previously published interaction studies establish that trichomonads can attach to several distinct surfaces; this ability could be important to successful parasitisation and thus explain the high incidence of trichomoniasis around the world. The large variety of structures involved in parasite adhesion, including proteins (Lehker \& Sweeney 1999, Mundodi et al. 2004), sugars (Mirhaghani \& Warton 1998) and lipophosphoglycans (Bastida-Corcuera et al. 2005), indicates that

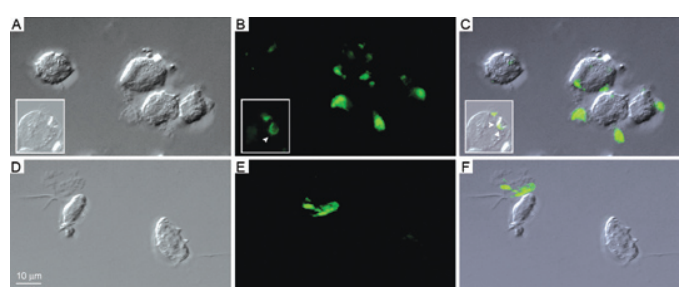

Fig. 4: Trichomonas vaginalis (A-C) and Trichomonas foetus (C, D) after interaction with human keratin. Immunofluorescence using a monoclonal anti-keratin antibody detected by Alexa Fluor 488-conjugated secondary antibody. Positive labelling is observed on fragments attached to T. vaginalis (A-C) and T. foetus (D-F). Inserts show a positively labelled endocytosed keratin fragment (arrowheads in A-C). A-D: differential imaging contrast system; B, E: Alexa green fluorescence; C, F: merge.
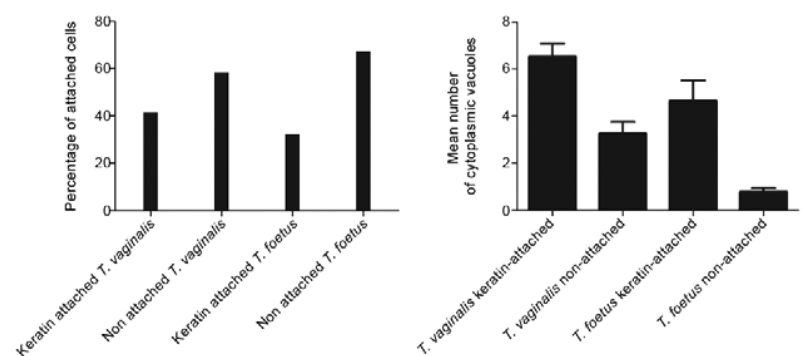

Fig 5: comparison between vacuoles found in Trichomonas vaginalis and Trichomonas foetus. Note that when parasites were attached to keratin, it was observed an increase in the number of vacuoles $(\mathrm{p}<0.031)$. 
trichomonads have the ability to adapt to various types of surfaces to establish infection. The ability of trichomonads to interact with keratin and ingest keratin particles could be very important for three reasons. First, keratin could be a source of protein and nutrition for the parasites. Second, this ability could enhance parasite access to the epithelial layer. Third, the adherence of trichomonads to VECs is important because parasites are transmitted during coitus; thus, the parasites could contact keratinised cells. In cattle, for example, breeding occurs only at oestrus, the time in the sexual cycle when keratinised squamous epithelial cell number is increasing (Roberts 1956). Our observations that parasites bound significantly to keratin indicate that there could be an opportunity for colonisation at this stage of the cycle, when more surface cells are keratinised. In a study of experimental infection in heifers, Parsonson et al. (1976) isolated T. foetus from the cervicovaginal mucus of 19 of the 20 cows bred to an infected bull. At necropsy, 15 of the 20 animals had vaginal infections, whereas some of them had infections of the uterus or oviducts. In all cases, $T$. foetus was found on the surface of the epithelium. Thus, adherence to VECs may result in vaginal colonisation and persistent infection, which is characteristic of trichomoniasis. This property could be very important and add more data to explain the success of these pathogens.

\section{REFERENCES}

Alderete JF, Garza GE 1985. Specific nature of Trichomonas vaginalis parasitism of host cell surfaces. Infect Immun 50: 701-708.

Alderete JF, Garza JE 1988. Identification and properties of Trichomonas vaginalis proteins involved in cytoadhrence. Infect Immun 56: 28-33.

Alvarez-Sánchez ME, Avila-González L, Becerril-García C, FattelFacenda LV, Ortega-López J, Arroyo R 2000. A novel cysteine proteinase (CP65) of Trichomonas vaginalis involved in cytotoxicity. Microb Pathog 28: 193-202.

Bastida-Corcuera FD, Okumura CY, Colocoussi A, Johnson PJ 2005. Trichomonas vaginalis lipophosphoglycan mutants have reduced adherence and cytotoxicity to human ectocervical cells. Eukaryot Cell 4: 1951-1958.

Benchimol M, Batista C, De Souza W 1990. Fibronectin and lamininmediated endocytic activity in the parasitic protozoa Trichomonas vaginalis and Tritrichomonas foetus. J Submicrosc Cytol Pathol 22: 39-45.

BonDurant R 2005. Venereal diseases of cattle: natural history, diagnosis and the role of vaccines in their control. Vet Clin North Am Food Anim Pract 21: 383-408.

Corbeil LB, Hodgson JL, Jones DW, Corbeil RR, Widders PR, Stephens LR 1989. Adherence of Tritrichomonas foetus to bovine vaginal epithelial cells. Infect Immun 57: 2158-2165.

Costa e Silva-Filho F, de Souza W, Lopes JD 1988. Presence of laminin-binding proteins in trichomonads and their role in adhesion. Proc Natl Acad Sci USA 85: 8042-8046.
Crouch ML, Alderete JF 1999. Trichomonas vaginalis interactions with fibronectin and laminin. Microbiology 145: 2835-2843.

Diamond LS 1957. The establishment of various trichomonads of animals and man inaxenic cultures. J Parasitol 43: 488-490.

Escario A, Gómez-Barrio A, Simons-Diez B, Escario JA 2009. Immunohistochemical study of the vaginal inflammatory response in experimental trichomoniasis. Acta Trop 14: 22-30.

Galvin SR, Cohen MS 2004. The role of sexually transmitted diseases in HIV transmission. Nat Rev Microbiol 2: 33-42.

Johnston VJ, Mabey DC 2008. Global epidemiology and control of Trichomonas vaginalis. Curr Opin Infect Dis 21: 56-64.

Kassem HH, Majoud OA 2006. Trichomoniasis among women with vaginal discharge in Benghazi city, Libya. J Egypt Soc Parasitol 36: 1007-1016.

Lehker MW, Sweeney D 1999. Trichomonad invasion of the mucous layer requires adhesins, mucinases and motility. Sex Transm Infect 75: 231-238.

Midlej V, Vilela R, Dias AB, Benchimol M 2009. Cytopathic effects of Tritrichomonas foetus on bovine oviduct cells. Vet Parasitol 12: 16-30.

Mirhaghani A, Warton A 1998. Involvement of Trichomonas vaginalis surface-associated glycoconjugates in the parasite/target cell interaction. A quantitative electron microscopy study. Parasitol Res 84: 374-381.

Mundodi V, Kucknoor AS, Klumpp DJ, Chang TH, Alderete JF 2004. Silencing the AP65 gene reduces adherence to vaginal epithelial cells by Trichomonas vaginalis. Mol Microbiol 53: 1099-1108.

Nanda N, Michel RG, Kurdgelashvili G, Wendel KA 2006. Trichomoniasis and its treatment. Expert Rev Anti Infect Ther 4: 125-135.

Parsonson IM, Clark BL, Dufty JH 1976. Early pathogenesis and pathology of Tritrichomonas foetus infection in virgin heifers. J Comp Pathol 86: 59-66.

Petrin D, Delgaty K, Bhatt R, Garber G 1998. Clinical and microbiological aspects of Trichomonas vaginalis. Clin Microbiol Rev 11: 300-317.

Rendon-Maldonado GJ, Espinosa-Cantellano M, Gonzalez-Robles A, Martinez-Palomo A 1998. Trichomonas vaginalis: in vitro phagocytosis of lactobacilli, vaginal epithelial cells, leukocytes and erytrocytes. Exp Parasitol 89: 241-250.

Rendon-Maldonado GJ, Espinosa-Cantellano M, Soler C, Torres JV, Martinez-Palomo A 2003. Trichomonas vaginalis: in vitro attachment and internalization of HIV-1 and HIV-1-infected lymphocytes. J Eukaryot Microbiol 50: 43-48.

Roberts SJ 1956. Veterinary obstetrics and genital diseases, Edwards Brothers Inc, Ann Arbor, 269 pp.

Schwebke JR, Burgess D 2004. Trichomoniasis. Clin Microbiol Rev 17: 794-803.

Wølner-Hanssen P, Krieger JN, Stevens CE, Kiviat NB, Koutsky L, Critchlow C, DeRouen T, Hillier S, Holmes KK 1989. Clinical manifestations of vaginal trichomoniasis. JAMA 27: 571-576. 